\title{
EMG-based Robot Control Interfaces: Past, Present and Future
}

\section{Panagiotis Artemiadis*}

School for Engineering of Matter, Transport and Energy, Ira A. Fulton Schools of Engineering, Arizona State University, USA

\begin{abstract}
Robots are now more than ever capable of performing a plethora of demanding tasks. However, as the presence of robotic technologies in everyday life grows rapidly, the role of humans in controlling the robots becomes of utmost importance. An efficient human-robot control interface is, therefore, required. In particular, an interface that would allow the user to control a robot in a continuous way by performing natural motions with his/her arm would be very effective. Neural interfaces and especially surface electromyographic (EMG) signals were proposed more than 30 years ago for actuating prosthetic limbs. Since then, a wide-range of algorithms and hardware solutions have been proposed. Multi-fingered prosthetic hands and agile arms are now capable of mimicking the function that the human counterparts provide. However, advances in the EMG-based control of prosthetics did not proceed at the same speed. This article will give an overview of the research done on the EMG-based human-robot control interfaces, focusing on the main advantages and disadvantages of this technology. Finally, future directions of the field will be discussed.
\end{abstract}

\section{Background \& Introduction}

Despite the fact, robots came to light approximately 50 years ago, the way humans control them is still an important issue. In particular, since the use of robots is increasingly widening to everyday life tasks (e.g. service robots, robots for clinical applications), the human-robot interface plays a role of the utmost significance. A large number of interfaces have been proposed in previous works, where some examples can be found in [1-3]. Most of the previous works propose complex mechanisms or systems of sensors, while in most of the cases the user should be trained to map his/her action (i.e. three dimensional (3D) motion of a joystick or a haptic device) to the resulted motion of the robot. When a human controls a robot, it is desirable to use natural limb movements or signals generated from him/her. This would improve the immediacy of the robot control as the human operator would consider the robot as extension of him/herself.

Neural interfaces for controlling robots have been proposed in applications ranging from prosthetics to remote teleoperation. Focusing on non-invasive interfaces, surface electromyography (EMG) has gained a lot of interest during the last decades. Surface EMG signals provide an excellent source of information of the user intention to move, therefore can be utilized as control interfaces for robots. They provide a fast and practical way of communicating control commands to artificial systems, since their recording requires surface placement of passive electrodes on the user's skin. Moreover, since EMG signals encode information that is generated by the brain, they can contain planning and execution information that can be very useful for the control of artificial systems. Up to the present, many researchers have investigated EMG signals as control interface for robots. The studies in this field can be classified into three groups: the control of prosthetic arms and hands; the application to orthoses mainly for rehabilitation purposes; and the control of remotely operated robots. As examples of the first, Smagt et al. [4] used EMG signals from ten muscles of the forearm to control a four-fingered robot hand. Myoelectric signals were also used for the control of a complex robot hand in Farry et al. [5]. A hand prosthesis using pattern recognition of myo-electric signals can be also found in Kato et al. [6]. Quite recently, Tenore F et al. [7] achieved to identify 12 individuated flexion and extension movements of the fingers using EMG signals from muscles of the forearm of an able-bodied subject, while a hydraulically driven multifunction prosthetic hand was driven by EMG signals in Schulz et al. [8]. A review on controlling prosthetic hands using EMG signals can be found in Zecca et al. [9] and Scott et al. [10]. The Boston Arm [11] and the Utah Arm [12] were driven by EMG signals in the past too.

Regarding orthotic devices, a lot of robotic mechanisms intended for either rehabilitation or extension of human ability have been developed during the last decades. As examples of the latter, Kazerooni proposed a new class of robot manipulator worn by humans in [13]. In these devices, the human in physical contact with the robotic manipulator exchange power and information signals. Similar orthotic devices for the upper limb have been developed and presented in [14]. In this case, the exoskeleton robot serving as an assistive device worn by the human, functions as a human-amplifier, while EMG signals are used as the main control signal providing high intuitiveness.

Robotic manipulators have been often remotely operated using EMG signals. Fukuda et al. [15] proposed a human-assisting manipulator teleoperated by EMG signals and arm motions. A position tracking system was used to track arm motions, while EMG signals recorded from the forearm muscles were used to estimate hand and wrist motion. A 2-dimensional myoelectric control of a robot arm was realized recently in [16].

In most of the studies mentioned earlier, EMG signals were used to decide the desired posture (in the cases of robot hands) or direction of motion (in the cases of prosthetic and remotely operated robot arms). However, a continuous representation of the user's motion would be essential for an effective EMG-driven robotic device. Particularly, in the case of robot arm control, a control interface able to output smooth profiles of motion, rather than a set of binary or generally discrete variables, is of utmost importance.

*Corresponding author: Panagiotis Artemiadis, School for Engineering of Matter, Transport and Energy, Ira A. Fulton Schools of Engineering, Arizona State University, USA, Tel: 480-965-4182, E-mail: panagiotis.artemiadis@asu.edu

Received July 24, 2012; Accepted July 26, 2012; Published July 28, 2012

Citation: Artemiadis P (2012) EMG-based Robot Control Interfaces: Past, Present and Future. Adv Robot Autom 1:e107. doi:10.4172/2168-9695.1000e107

Copyright: $\odot 2012$ Artemiadis P. This is an open-access article distributed unde the terms of the Creative Commons Attribution License, which permits unrestricted use, distribution, and reproduction in any medium, provided the original author and source are credited. 
Artemiadis and colleagues have worked on the EMG based control of robots over the last 8 years and provided a wide range of algorithmic approaches that decode robot control variables from EMG signals. A novel decoding model that can estimate a continuous representation of the human upper limb motion through multiple EMG channels has been proposed [17]. Moreover, a method that detects and compensates for changes of EMG recordings due to muscle fatigue has also been proposed proposed and tested [18-20]. The description of anthropomorphic arm movement and its fusion to the EMG decoding algorithm has been shown to improve decoding results in [21] and [22].

\section{Why EMG?}

Since EMG signals seem rather informative but difficult to decode and use for the control of devices, a list of specific advantages and disadvantages would be worth analyzing.

\section{Advantages}

Recording: In order to record EMG signals, surface passive electrodes need to be placed on specific locations on the limb. For arm muscles, it is generally easy to find the placement location of the electrodes, using the related literature [23]. Current technology allows the onsite (i.e. in the electrode) processing and amplification of the signal, as well as the wireless transmission of the signal to a remotely located station, a feature that significantly increases the practicality and efficiency of the recording [24].

Prediction: In most skeletal muscles, the EMG activity precedes the motion of the actuated limb. Activation and musculoskeletal dynamics are the main sources of this Electro Mechanical Delay (EMD), which is at the range of 50-100 ms based on the actual muscles. In other words, EMG signals can give information about the intention of motion, 50$100 \mathrm{~ms}$ before the motion actually happens. This feature can be greatly used in interfaces where such time delays in action can play significant role, e.g. for flight controllers.

Range of information: Skeletal muscles are not only responsible for moving our limbs. They are used to exert force to the environment, or withstand external forces, by adjusting the limb impedance. In fact, our musculoskeletal structure is capable of controlling the motion and the impedance of the limb independently, a feature that was only recently accomplished by robots. EMG is the only interface that can give information about the human limb impedance, without mechanically perturbing the arm. Finally, EMG recordings can also give information about the muscle fatigue, which can be related to the overall physiological condition of a human subject during the execution of a task. This information can be very useful in artificial systems working together with humans.

\section{Disadvantages}

Non-stationarity: EMG signals correspond to the activation of muscle fibers, however recorded over layers of muscle, fat and tissue. This highly affects the features of the recorded signal, which can alter significantly by various reasons, e.g. changes of the electrode position, sweat at the recording site, changes of the impedance of the electrode, muscle fatigue etc. Although Artemiadis et al. have proposed methods for compensating for those changes [18-20], more effort has to be made for achieving robust recordings, both in hardware and software level.

Subject-specificity: EMG signals are physiological signals, and as most of the latter, they vary from subject to subject. The subject's physical condition as well as many other parameters can significantly affect the muscle activation recordings. The systems proposed so far include a training period where EMG recordings are being input into machine learning and pattern recognition methods in order to train a mathematical model to decode motion information.

Crosstalk: The human musculoskeletal system is highly redundant in the level of actuation. In fact, more than 30 muscles actuate 7 degrees of freedom in the human upper limb, excluding finger motion. Muscles are very close to each other, and in most of the cases overlapped inside our limbs. Therefore, recording from a specific muscle is not always easy. Crosstalk between adjacent muscles is almost always present, especially in the muscles for the upper limb. Therefore, recorded EMG signals do not convey information only from a specific muscle.

\section{Conclusion \& Future Directions}

As neural interfaces are gaining interest in different fields ranging from medical applications (motor rehabilitation, neuroprosthetics, assistive devices) to human computer interfaces (gaming technology, remotely operated devices, human-machine interaction), EMG signals play a significant role due to their practicality and noninvasiveness. It is, however, the efficiency of this human machine control interface that will finally decide whether they are going to be used in every-day-life applications or not. Advances both in hardware (smart electrodes, on board computing, etc) and software (auto-calibration, high-bandwidth information decoding, etc) are required for making EMG an efficient control interface. It is the author's belief that the research community is not far from making those advancements reality, and that EMG-based control of artificial systems will revolutionize the 2010 decade.

\section{References}

1. Woo-Keun Y, Goshozono T, Kawabe H, Kinami M, Tsumaki Y, et al.(2004) Model-based space robot teleoperation of ETS-VII manipulator. IEEE Trans on Robotics and Automation 20: 602-612.

2. Tayh-Jong T, Bejczy AK, Chuanfan G, Ning X (1994) Intelligent planning and control for telerobotic operations. Proc of IEEE/RSJ/GI Int Conf Intelligent Robots and Systems 1: 389-396.

3. Park J, Khatib O (2006) A haptic teleoperation approach based on contact force control. Int J Rob Res 25: 575-591.

4. Bitzer S, van der Smagt P (2006) Learning EMG control of a robotic hand towards active prostheses. Proc of IEEE Int Conf on Robotics and Automation 2819-2823.

5. Farry KA, Walker ID, Baraniuk RG (1996) Myoelectric teleoperation of a complex robotic hand. IEEE Trans. On Robotics and Automation 12: 775-788.

6. Kato I, Okazaki E, Kikuchi H, Iwanami K (1967) Electropneumatically controlled hand prosthesis using pattern recognition of myo-electric signals, in Dig. 7th ICMBE 367

7. Tenore F, Ramos A, Fahmy A, Acharya S, Etienne-Cummings R, et al. (2007) Towards the control of individual fingers of a prosthetic hand using surface EMG signals. Proc 29th Annual Int Conf of the IEEE EMBS 6145-6148.

8. Schulz S, Pylatiuk C, Reischl M, Martin J, Mikut R, et al. (2005) A hydraulically driven multifunctional prosthetic hand. Robotica 23: 293-299.

9. Zecca M, Micera S, Carrozza MC, Dario P (2002) Control of multifunctional prosthetic hands by processing the electromyographic signal. Crit Rev Biomed Eng 30: 459-485.

10. Scott RN, Parker PA (1988) Myoelectric prostheses: state of the art. J Med Eng Technol 12: 143-151.

11. Jerard RB, Williams TW, Ohlenbusch CW (1974) Practical design of an EMG controlled above elbow prosthesis. Proc of Conf Engineering Devices for Rehabilitation 73. 
Citation: Artemiadis P (2012) EMG-based Robot Control Interfaces: Past, Present and Future. Adv Robot Autom 1:e107. doi:10.4172/21689695.1000e107

Page 3 of 3

12. Jacobson SC, Knutti DF, Johnson RT, Sears HH (1982) Development of the utah artificial arm. IEEE Trans Biomed Eng 29: 249-269.

13. Kazerooni $H$ (1990) Human-robot interaction via the transfer of power and information signals. IEEE Trans. Syst Man Cybern 20: 450-463.

14. Cavallaro E, Rosen J, Perry JC, Burns S, Hannaford B (2005) Hill-based model as a myoprocessor for a neural controlled powered exoskeleton arm - parameters optimization. Proc of IEEE Int Conf on Robotics and Automation 4514-4519.

15. Fukuda O, Tsuji T, Kaneko M, Otsuka A (2003) A human assisting manipulato teleoperated by EMG signals and arm motions. IEEE Trans on Robotics and Automation 19: 210-222.

16. Celani NML, Soria CM, Orosco EC, di Sciascio FA, Valentinuzzi ME (2007) Two-dimensional myoelectric control of a robotic arm for upper limb amputees. J Phys Conf Ser 90.

17. Artemiadis PK, Kyriakopoulos KJ (2010) EMG-based control of a robot arm using low-dimensional embeddings. IEEE Transactions on Robotics 26: 393 398

18. Artemiadis PK, Kyriakopoulos KJ (2010) An EMG-based robot control scheme robust to time-varying emg signal features. IEEE Transactions on Information Technology in Biomedicine14: 582-588.

19. Artemiadis PK, Kyriakopoulos KJ (2011) A switching regime model for the EMG-based control of a robot arm. IEEE Transactions on Systems, Man, and Cybernetics, Part B: Cybernetics 41: 53-63.

20. Artemiadis PK, Kyriakopoulos KJ (2008) Assessment of muscle fatigue using a probabilistic framework for an EMG-based robot control scenario. Proc of IEEE Int Conf Bioinformatics and Bioengineering 1-6.

21. Artemiadis PK, Kyriakopoulos KJ (2006) EMG-based teleoperation of robot arm in planar catching movements using ARMAX model and trajectory monitoring techniques. Proc of IEEE Int Conf on Robotics and Automation 3244-3249.

22. Artemiadis PK, Kyriakopoulos KJ (2009) A bio-inspired filtering framework fo the EMG-based control of robots. Proc of 17th Mediterranean Conference on Control and Automation 1155-1160.

23. Cram JR, Kasman GS (1998) Introduction to Surface Electromyography. Inc Gaithersburg, Maryland: Aspen Publishers.

24. Delsys[WWW]. Available from: http://www.delsys.com[Accessed 25/7/12] 